\title{
Surface-Passivated AIGaN Nanowires for Enhanced Luminescence of Ultraviolet Light Emitting Diodes
}

Haiding Sun, Mohammad Khaled Shakfa, Mufasila Muhammed, Bilal Janjua, Kuanghui Li, Ronghui Lin, Tien Khee Ng, Iman S Roqan, Boon S. Ooi, and Xiaohang Li

ACS Photonics, Just Accepted Manuscript • DOI: 10.1021/acsphotonics.7b01235 • Publication Date (Web): 19 Dec 2017

Downloaded from http://pubs.acs.org on December 24, 2017

\section{Just Accepted}

"Just Accepted" manuscripts have been peer-reviewed and accepted for publication. They are posted online prior to technical editing, formatting for publication and author proofing. The American Chemical Society provides "Just Accepted" as a free service to the research community to expedite the dissemination of scientific material as soon as possible after acceptance. "Just Accepted" manuscripts appear in full in PDF format accompanied by an HTML abstract. "Just Accepted" manuscripts have been fully peer reviewed, but should not be considered the official version of record. They are accessible to all readers and citable by the Digital Object Identifier (DOI®). "Just Accepted" is an optional service offered to authors. Therefore, the "Just Accepted" Web site may not include all articles that will be published in the journal. After a manuscript is technically edited and formatted, it will be removed from the "Just Accepted" Web site and published as an ASAP article. Note that technical editing may introduce minor changes to the manuscript text and/or graphics which could affect content, and all legal disclaimers and ethical guidelines that apply to the journal pertain. ACS cannot be held responsible for errors or consequences arising from the use of information contained in these "Just Accepted" manuscripts. 


\title{
Surface-Passivated AlGaN Nanowires for Enhanced Luminescence of Ultraviolet Light Emitting Diodes
}

Haiding Sun ${ }^{1}$, Mohammad Khaled Shakfa ${ }^{2}$, Mufasila Mumthaz Muhammed ${ }^{3}$, Bilal Janjua ${ }^{2}$, Kuang-Hui Li ${ }^{1}$,

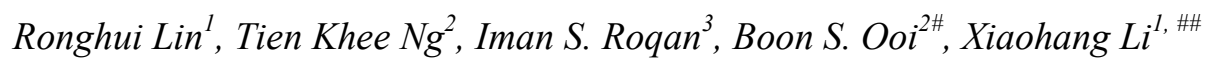

${ }^{1}$ King Abdullah University of Science \& Technology (KAUST), Computer, Electrical, and Mathematical Sciences and Engineering Division, Advanced Semiconductor Laboratory, Thuwal 23955-6900, Saudi Arabia

${ }^{2}$ King Abdullah University of Science \& Technology (KAUST), Computer, Electrical, and Mathematical Sciences and Engineering Division, Photonics Laboratory, Thuwal 23955-6900, Saudi Arabia

${ }^{3}$ King Abdullah University of Science and Technology (KAUST), Physical Sciences and Engineering Division, Spectroscopy and Semiconductor Research Laboratory, Thuwal 23955, Saudi Arabia

\section{KEYWORDS}

Aluminum gallium nitride nanowire, passivation, ultraviolet light emitting diode, potassium hydroxide, surface oxides and states, surface recombination

\begin{abstract}
Spontaneously-grown, self-aligned AlGaN nanowire ultraviolet light emitting diodes still suffer from low efficiency partially because of the strong surface recombination caused by surface states, i.e., oxidized surface and high density surface states. Several surface passivation methods have been introduced to reduce surface non-radiative recombination by using complex and toxic chemicals. Here, we present an effective method to suppress such undesirable surface recombination of the $\mathrm{AlGaN}$ nanowires via diluted potassium hydroxide $(\mathrm{KOH})$ solution; a commonly used chemical process in semiconductor fabrication which is barely used as surface passivation solution in self-assembled nitride-based nanowires. The transmission electron microscopy investigation on the samples reveals almost intact nanowire structures after the passivation process. We demonstrated an approximately $49.7 \%$ enhancement in the ultraviolet light output power after 30-s $\mathrm{KOH}$ treatment on AlGaN nanowires grown on titanium-coated silicon substrates. We attribute such a remarkable enhancement to the removal of the surface
\end{abstract}


ACS Photonics Revised Manuscript \# ph-2017-01235t

dangling bonds and oxidized nitrides (Ga-O or Al-O bonds) at the surface as we observe the change of the carrier lifetime before and after the passivation. Thus, our results highlight the possibility of employing this process for the realization of high performance nanowire UV emitters. 
Ultraviolet light emitting diodes (UVLEDs) are crucial for a number of applications such as water/air/food sterilization, free-space non-line-of-sight communication and various medical applications. ${ }^{1,2}$ The bandgap of $\mathrm{Al}_{\mathrm{x}} \mathrm{Ga}_{1-\mathrm{x}} \mathrm{N}(0 \leq \mathrm{x} \leq 1)$ alloys can be tuned from 3.4 to $6.1 \mathrm{eV}$ by changing the alloy composition which covers the entire UV spectral region from 360 to $210 \mathrm{~nm}$. This makes AlGaN alloys particularly suitable for the realization of UVLEDs. ${ }^{3}$ Nevertheless, the development of UVLEDs performance has not yet reached the level achieved in the visiblespectrum LEDs. Despite intense efforts to improve the performance of AlGaN-based UVLEDs, the existing AlGaN-based planar UVLEDs suffer from high threading dislocation densities in the AlGaN epitaxial layer, limited light extraction channels, poor p-type doping efficiency, and difficulties in heat dissipation under high current injection. ${ }^{4,5,6}$ As a result, the maximum external quantum efficiency (EQE) of fully packaged AlGaN-based UVLEDs, in the entire UV spectral region (360 to $210 \mathrm{~nm}$ ), is extremely low ( $\leq 15 \%)$. However, recently, Takano et al. claimed achieving a maximum EQE of $20 \%$ of EQE for a $275 \mathrm{~nm} \mathrm{LED.}{ }^{1}$ These EQE values are much lower than that obtained in highly commercialized InGaN-based blue LEDs, the EQE efficiency of which can be as high as $80 \%{ }^{7}$ Thus, developing the conventional planar UVLED structure to obtain high EQE remains tremendously challenging. Recently, the AlGaN nanowires (NWs) emerged as an alternative approach for the realization of UVLEDs and UV laser diodes. AlGaN nanowire structure possesses many advantages relative to planar structures. In particular, because of the effective lateral stress relaxation during growth, nearly defect-free $\mathrm{AlGaN}$ nanowires can be formed directly on silicon or other foreign substrates (such as metal and graphene) with high internal quantum efficiency. ${ }^{8,9}$ Moreover, the p-type doping efficiency in AlGaN has been significantly improved due to the reduced activation energy of $\mathrm{Mg}$ dopant in nanowire structures. ${ }^{10}$ In addition, the emitted photons can easily escape and thus enhance the light extraction efficiency owing to the large surface-to-volume ratio and the multiple scattering effects in nanowire structures. ${ }^{11}$ Consequently, by adopting AlGaN nanowires instead of planar structures, researchers were able to achieve an electrically-pumped deep UV laser emitting at a very short wavelength of $239 \mathrm{~nm}^{12}$ and attain a droop-free UVLED on metal substrates. ${ }^{13}$

Even though significant progress has been made in the past few years, the development of $\mathrm{AlGaN}$ nanowire-based emitters is at its early stage. Due to the large surface-to-volume ratio of nanowire structures, for all nitride-based devices, the optical and electrical properties of these 
structures are highly sensitive to their surroundings. For example, a significant presence of surface states, surface Fermi level pinning effects, and an enhanced surface recombination due to the Shockley-Read-Hall(SRH) non-radiative recombination at these surface state/defect centers will lead to the light emitting devices of low efficiency. Significant efforts have been dedicated to the development of methodologies aimed at removing such surface defects (such as dangling bonds) and suppressing surface recombination to improve the performance of the nitride-based nanowire devices. ${ }^{14,15,16,17}$ Zhao et al. achieved success in mitigating SRH recombination by octadecylthiol (ODT) and sulfide passivation process in InGaN/GaN nanowires, resulting in an improved device efficiency. ${ }^{16}$ Hetzl et al. introduced a self-regulated AlGaN shell on GaN nanorod as a mean of passivating defect-related recombination centers. ${ }^{17} \mathrm{Mi}$ et al. investigated the use of inorganic sulfides, such as $\left(\mathrm{NH}_{4}\right)_{2} \mathrm{~S}_{\mathrm{X}}$, for nanowire surface passivation. They found that such sulfides can reduce the surface recombination velocity and hence improve the LED performance. ${ }^{18}$ In addition, $\mathrm{Si}_{4} \mathrm{~N}_{3}$ and $\mathrm{SiO}_{2}$ were used to passivate GaN surface. ${ }^{19}$ All of these passivation approaches, while using different chemicals, demonstrated the effectiveness of this process in improving the device performance. However, in these studies, toxic and hazardous chemicals were used or their techniques required an additional material deposition procedure to passivate the nanowires.

In this paper, we propose the use of diluted potassium hydroxide $(\mathrm{KOH})$ solution to passivate our spontaneously grown $\mathrm{AlGaN}$ nanowires prior to device fabrication. The $\mathrm{KOH}$ solution is commonly used as a follow-up step after dry etching of GaN-based thin films to improve the sidewall roughness for light emitting devices ${ }^{20,21}$ or in the fabrication of GaN-based metal-insulator-semiconductor power devices. ${ }^{22}$ Here, we demonstrate that $\mathrm{KOH}$ can also be used as a surface passivation solution. We investigate the underlying passivation mechanism and its effects on the optical properties of AlGaN nanowires. We study the effect of the $\mathrm{KOH}$ passivation processes of different durations on the optical performance of these AlGaN nanowires, compared to that unpassivated nanowires.

\section{METHODS}

The AlGaN nanowire LED structures studied in this work were grown on n-type $\mathrm{Si}(001)$ coated with a 100-nm Ti film by e-beam evaporator using Veeco Gen 930 plasma-assisted 
molecular beam epitaxy (PAMBE) under nitrogen-rich conditions. A 2-step growth process was implemented. The nanowires were first nucleated at relatively low temperature of $\sim 500{ }^{\circ} \mathrm{C}$, followed by the growth of the AlGaN layers at a higher temperature of $\sim 630{ }^{\circ} \mathrm{C}$. The nominal $\mathrm{Al}$ composition was estimated by taking the ratio of $\mathrm{Al}$ to the total metal beam equivalent pressure as measured by the beam flux monitor. To fill the gap between nanowires, we used parylene-C, consisting of a deposition step followed by the etch-back process to reveal the p-GaN contact layers. More details of the growth conditions and fabrication process can be found in our earlier work. ${ }^{13}$ The p-i-n nanowire LED started with a $100-\mathrm{nm}$ n-GaN layer, followed by $\sim 50-\mathrm{nm}$ Sidoped $\mathrm{Al}_{0.3} \mathrm{Ga}_{0.7} \mathrm{~N}$ layer and then the active region of 30 -nm-thick $\mathrm{Al}_{0.14} \mathrm{Ga}_{0.86} \mathrm{~N}$ layers. Afterwards, a $\sim 50 \mathrm{~nm} \mathrm{Mg-doped} \mathrm{Al}_{0.3} \mathrm{Ga}_{0.7} \mathrm{~N}$ layer was grown and the structures completed with $\mathrm{a} \sim 20 \mathrm{~nm} \mathrm{Mg-doped} \mathrm{GaN} \mathrm{layer} \mathrm{as} \mathrm{the} \mathrm{p-type} \mathrm{contact} \mathrm{layer.} \mathrm{After} \mathrm{the} \mathrm{growth,} \mathrm{the} \mathrm{nanowires} \mathrm{were}$ treated in a $\mathrm{KOH}$ solution with a concentration $10 \mathrm{wt} . \% \mathrm{KOH}$ at $45^{\circ} \mathrm{C}$ for different durations from 10 to $40 \mathrm{~s}$. After passivation, the nanowires were rinsed in deionized water for $10 \mathrm{mins}$. The nanowire LEDs were then fabricated immediately using the standard UV contact lithography process. Ni $(5 \mathrm{~nm}) / \mathrm{Au}(5 \mathrm{~nm})$ were deposited directly on the top of the p-GaN layer, which forms an ohmic contact with $\mathrm{p}-\mathrm{GaN}$, upon annealing at $600{ }^{\circ} \mathrm{C}$ under $\mathrm{O}_{2}$ gas ambient for $1 \mathrm{~min}$. $\mathrm{Ni}(10 \mathrm{~nm}) /$ Gold $(\mathrm{Au})(500 \mathrm{~nm})$ was then deposited as the top p-contact for probing. For $\mathrm{n}$ contact, a thickness of $\sim 100 \mathrm{~nm}$ of silicon was etched from the back side of the sample to expose a clean surface. $\mathrm{Ti}(10 \mathrm{~nm}) / \mathrm{Au}(150 \mathrm{~nm})$ were then sputtered as an n-pad followed by annealing in $\mathrm{N}_{2}$ gas ambient at $250{ }^{\circ} \mathrm{C}$ for $1 \mathrm{~min}$ to form an n-type ohmic contact. Room temperature photoluminescence (PL) measurements were performed using a $266 \mathrm{~nm}$ excitation pulse laser (SNU-20F-100). The PL signal was collected by using a UV objective and then measured by the OceanOptics QEPro spectrometer. Electroluminescent (EL) signal was also measured and the current was injected by a Keithley source $2450 \mathrm{C}$ operating in continuous mode under different injection currents. Scanning electron microscopy (SEM) and scanning transmission electron microscopy (STEM) measurements were performed to analyze the nanowire structures before and after passivation. The TEM specimens were prepared by using an FEI Helios dual-beam focused ion beam scanning electron microscope (DBFIB-SEM) system with a Ga ion source. The TEM microscope (Titan 80-300 ST) was operated at the accelerating voltage of $300 \mathrm{kV}$. Atomic-number sensitive (Z-contrast) STEM was realized by acquiring the data with high-angle annular dark-field (HAADF) detector. The high-resolution X-ray photoelectron spectroscopy 
(HRXPS) measurements were carried out using a Kratos Axis Ultra DLD spectrometer equipped with a monochromatic $\mathrm{Al} \mathrm{K} \alpha \mathrm{X}$-ray source $(\mathrm{h} v=1486.6 \mathrm{eV})$ operating at $150 \mathrm{~W}$, a multichannel plate, and delay line detector under a vacuum of $\sim 10^{-9}$ mbar. The high-resolution spectra were collected within the limits of spatial resolution at a fixed analyzer pass energy of $20 \mathrm{eV}$. The remnant binding energy shifts were referenced to the adventitious carbon (C 1s) signal. Timeresolved PL (TRPL) measurements were performed using a frequency-tripled Ti:Sapphire laser $(293 \mathrm{~nm})$ with a pulse width of $150 \mathrm{fs}$ and a synchro-scan streak-camera system. The overall temporal resolution of our setup is 2 ps. The average excitation power on the sample surface was maintained to be $1 \mathrm{~mW}$ at a $76 \mathrm{MHz}$ repetition rate.

\section{RESULTS AND DISCUSSION}
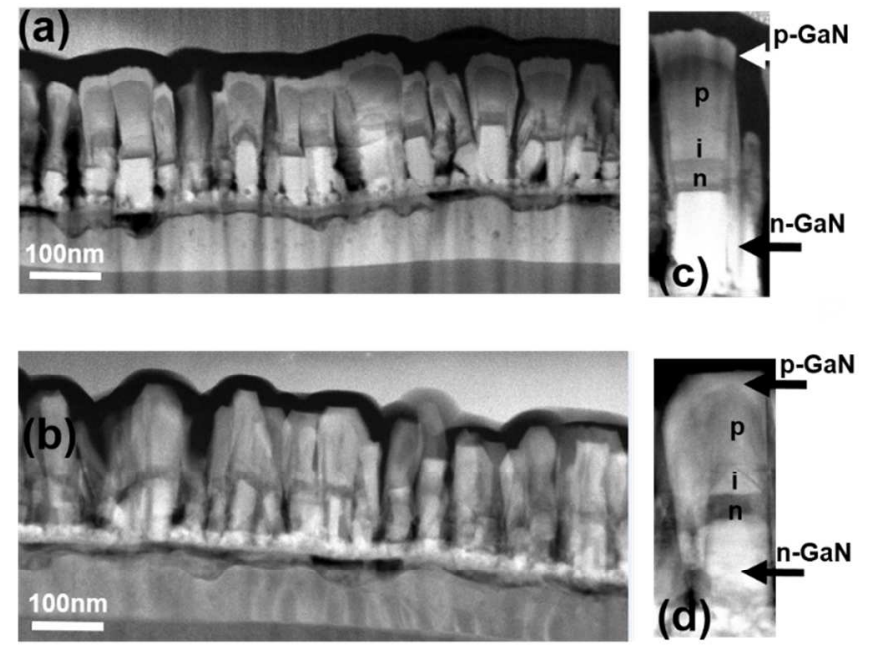
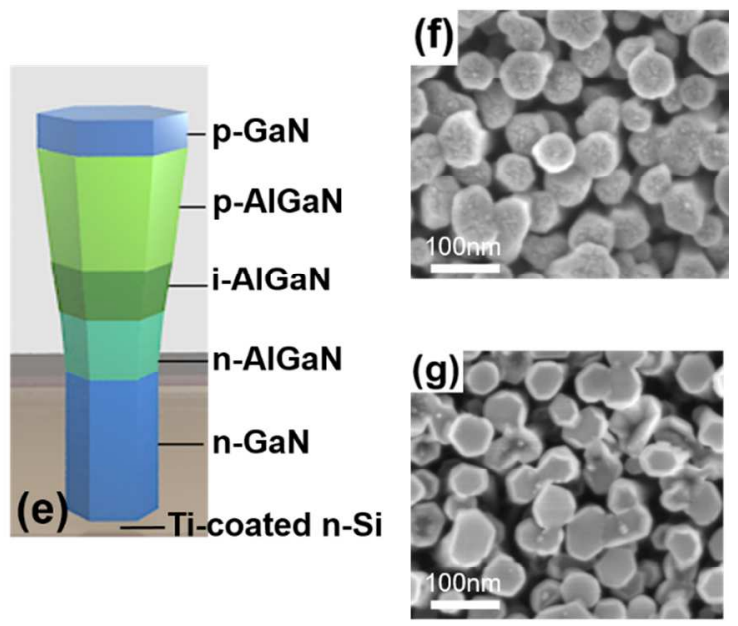

Figure 1. Structural characterization of the $\mathrm{AlGaN}$ nanowires before and after $\mathrm{KOH}$ passivation process. (a) and (b) Cross-sectional STEM-HAADF images show the vertically aligned nanowires before and after 30-s passivation, respectively. (c) and (d) Cross-sectional STEMHAADF images show a single nanowire before and after passivation, respectively. Each nanowire includes a bottom n-GaN layer, n-type $\mathrm{AlGaN}$ layer, i-AlGaN active layer, p-type AlGaN layer, and top p-GaN layer. (e) 3D schematics of the studied nanowire LEDs grown on $\mathrm{Ti} / \mathrm{Si}$ template substrate. (f) and (g) Top view SEM images of the AlGaN nanowires show tightly packed nanowires before and after passivation, respectively. 
Fig. 1 (a) and (b) show the cross-sectional STEM-HAADF images of the compact and nearly vertically aligned nanowires nucleated on Ti-coated Si (100) substrates before and after $\mathrm{KOH}$ Overall, after $30 \mathrm{~s}$ passivation, the nanowires remained almost intact. From the enlarged images shown in Fig. 1(c) and (d), we observe each layer in the nanowires along the growth direction, which is also schematically depicted in Fig. 1(e). We notice that the nanowire surface flattened after $\mathrm{KOH}$ passivation (see Fig. 1(d)). The surface flattening is further confirmed by comparing the top-view SEM images of the nanowires shown in Fig. 1(f) and 1(g). This phenomenon may potentially be attributed to the $\mathrm{KOH}$ etching of the top $\mathrm{p}-\mathrm{GaN}$ contact layer during the passivation process. Even though a 5-10 nm p-GaN was etched away, most of the p$\mathrm{GaN}$ layer still remained at the top part of the nanowires after immersion in the $\mathrm{KOH}$ solution for 30 -s.

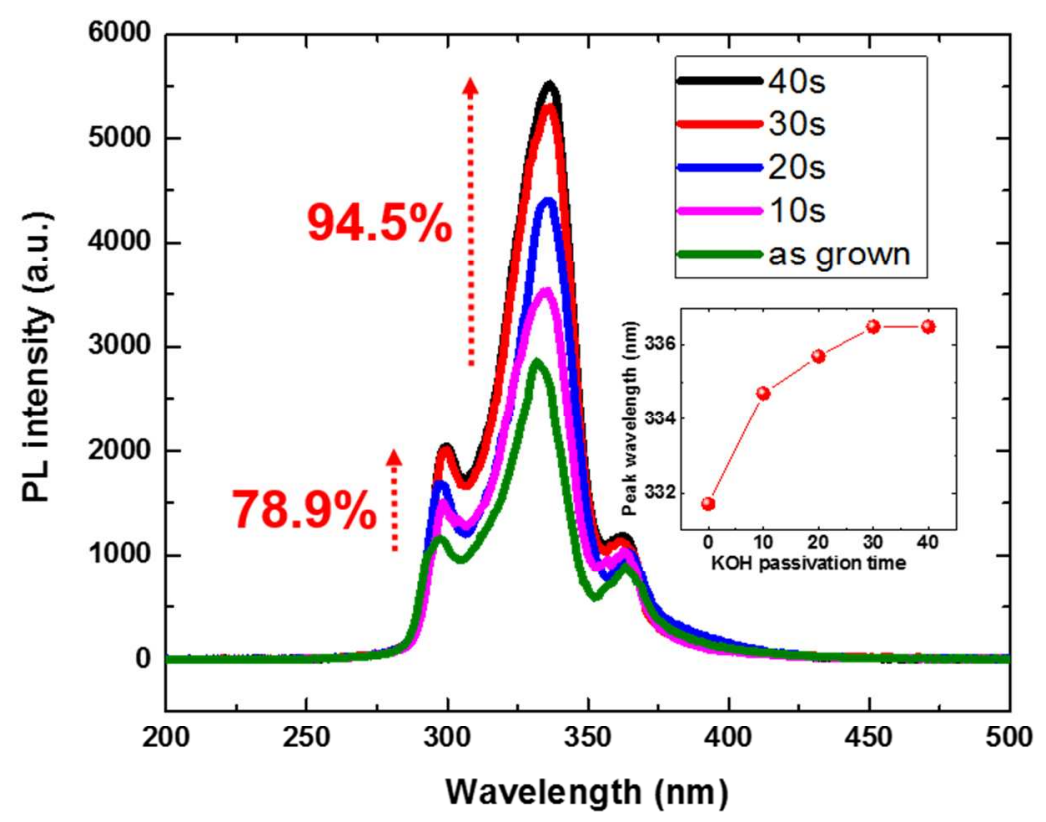

Figure 2. Room temperature PL spectra of the as-grown sample and the samples after 10, 20, 30, 40-s KOH passivation.

PL spectra of the nanowires pertaining to different $\mathrm{KOH}$ passivation durations are shown in Fig. 2. Three distinct peaks are observed in the PL spectrum of the as-grown sample (before passivation): the left shoulder of the major peak emitting at $296 \mathrm{~nm}$ arises from the higher Al- 
content $\mathrm{AlGaN}$ layer ( $\mathrm{n}$ and $\mathrm{p}-\mathrm{Al}_{0.3} \mathrm{Ga}_{0.7} \mathrm{~N}$ layer), the most intense peak of $332 \mathrm{~nm}$ originates from the active layer, and the right shoulder of the major peak emitting around $360 \mathrm{~nm}$ is related to the PL emission from the top and bottom GaN layer. As the passivation time is increased from 10 to $40 \mathrm{~s}$, it is evident the left shoulder peak and the major peak exhibit a slight redshift of 3.3 and $4.8 \mathrm{~nm}$, respectively. Most importantly, after $40 \mathrm{~s}$ passivation, the intensity of the peaks located at $296 \mathrm{~nm}$ and $332 \mathrm{~nm}$ increases by $78.9 \%$ and $94.5 \%$, respectively. We did not extend the passivation time further, as the PL enhancement after $40 \mathrm{~s}$ was not significant (as shown in the inset of Fig. 2). During the growth, the AlGaN shell can be formed naturally as a result of the diffusion-controlled growth mechanism of III-nitride nanowires under nitrogen-rich conditions. ${ }^{8}$ Due to the limited diffusion of $\mathrm{Al}$ atoms with respect to $\mathrm{Ga}$ (Al atoms have a shorter diffusion mean free path), a large amount of Al atoms may accumulate near the nanowire sidewalls, resulting in the formation of an Al-rich $\mathrm{Al}_{\mathrm{x}} \mathrm{Ga}_{1-\mathrm{x}} \mathrm{N}$ shell on the nanowire lateral surfaces. ${ }^{12,23}$ Due to the formation of the core-shell structure, after the $\mathrm{KOH}$ passivation, the Al-rich AlGaN shell might be etched which could lead to the observation of the redshift. This observation is consistent with the result reported by Martin et al. in which they observed a blueshift by adding an AlGaN shell to the GaN rods. ${ }^{17}$ In our case, since we partially removed Al-rich shell layer after passivation, the redshift would be expected. We believe the subject of the redshift mechanism after passivation is worthy of a separated detailed investigation, for example, by using nanoscale Cathodoluminescence and TEM, as reported by Aditya et al. ${ }^{24}$

There are various explanations for such PL emission enhancement after sample passivation. A flattened top surface of the nanowire indicated that we have possibly removed a portion $(\sim 5-10 \mathrm{~nm})$ of the p-GaN layer. Since $\mathrm{GaN}$ is rather absorptive in the UV region, this removal of a few nm of GaN could affect the total amount of escaped photons. To exclude this possibility, we grew an AlGaN nanowire LED without p-GaN and capped the nanowire with only a high $\mathrm{Al}$ content p-type AlGaN layer on top of the active region. Then, we carried out the passivation process with identical $\mathrm{KOH}$ solution and collected a series of PL spectra from these samples. On average, we still observe a strong enhancement (of $\sim 60.1 \%$ ) of the PL intensity with a noticeable redshift in the PL peak. This observation further confirms that the partial removal of $\mathrm{p}-\mathrm{GaN}$ is not the only factor contributing to the observed PL intensity enhancement. Additionally, the $\mathrm{KOH}$ treatment could also remove part of bottom $\mathrm{n}-\mathrm{GaN}$ and thus contribute to the increase 
of the PL intensity. To further explore possible reasons for the PL intensity enhancement due to $\mathrm{KOH}$ passivation, we performed a surface analysis of the nanowires using an HRXPS. In this study, to verify the possible changes in surface status, the Ga $2 p$, Ga $3 d$, and $\mathrm{Al} 2 p$ core levels were investigated for the analysis before and after passivation process. Comparing the peak positions of Ga $2 p$, Ga $3 d$, and $\mathrm{Al} 2 p$ core levels, this allowed us to verify the bonding status of these elements in the as-grown sample and the samples after $\mathrm{KOH}$ passivation. Fig. 3 shows the acquired core-level spectra of the Ga $2 p$, Ga $3 d$, and $\mathrm{Al} 2 p$ before and after $40 \mathrm{~s}$ passivation. Before passivation, the Ga $2 p$, Ga $3 d$, and $\mathrm{Al} 2 p$ peaks located at $1117.7 \pm 0.1,19.9 \pm 0.1$ and $74.4 \pm 0.1 \mathrm{eV}$, which is ascribed to the $\mathrm{Ga}-\mathrm{O}, \mathrm{Ga}-\mathrm{O}$, and $\mathrm{Al}-\mathrm{O}$ bonding, respectively, as shown in Fig. $3 \mathrm{a}^{25}$ After passivation, the peak positions of $\mathrm{Ga} 2 p$, Ga $3 d$ and $\mathrm{Al} 2 p$ shift to lower binding energy values of $1117.0 \pm 0.1,19.3 \pm 0.1$ and $73.1 \pm 0.1 \mathrm{eV}$, which is ascribed to the Ga-N, Ga-N and Al-N bonding. The shift in the binding energy associated with Ga and $\mathrm{Al}$ in the Ga $2 p$, Ga $3 d$ and $\mathrm{Al} 2 p$ core level spectra is a clear evidence of the removal of surface oxides due to $\mathrm{KOH}$ passivation. ${ }^{25}$ This observation is also consistent with the findings of an earlier study, in which researchers observed a reduction in the surface chemical composition ratio of Ga to $\mathrm{N}$ (from 2.68 to 1.73 ) in a three-dimensional GaN nano-cone array after the $\mathrm{KOH}$ treatment, resulting in an enhancement in the PL intensity by $60 \% .{ }^{26}$ In addition, the texturing surface of GaN nano-pillars by subjecting them to the $\mathrm{KOH}$ treatment could also improve the light extraction efficiency reflected in an enhancement in the PL. ${ }^{27}{ }^{28}$ Via the $\mathrm{KOH}$ post-treatment, The PL enhancement was ascribed to the alleviation of some of the surface damage in the formation of nano-cones or nano-pillars through the top-down fabrication. In our study, we reveal, for the first time, that $\mathrm{KOH}$ solution can also be used in the fabrication of self-assembled AlGaN nanowire-based devices. 

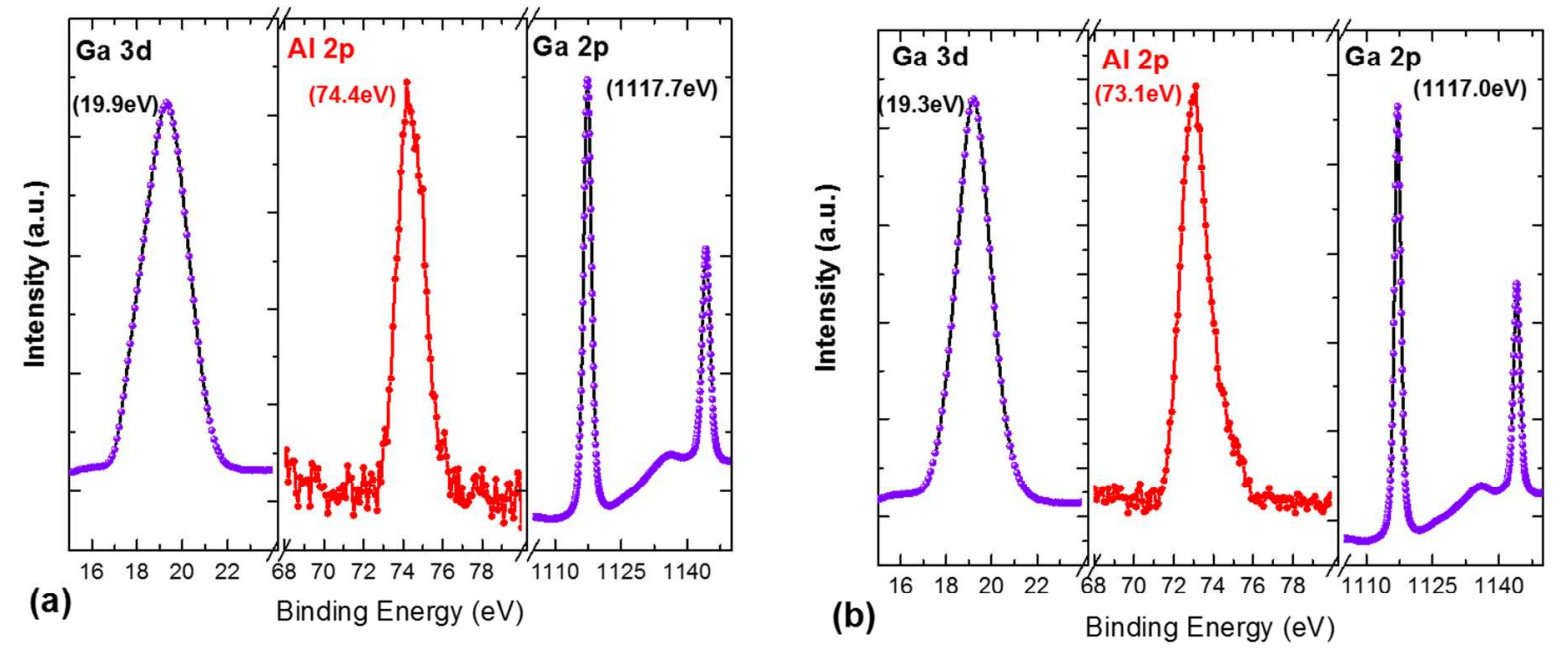

Figure 3. (a) and (b) Ga 2p, Ga $3 \mathrm{~d}$ and $\mathrm{Al} 2 \mathrm{p}$ core-level spectra before and after $40-\mathrm{s} \mathrm{KOH}$ passivation, respectively. The peak positions of core levels are shown in parentheses.
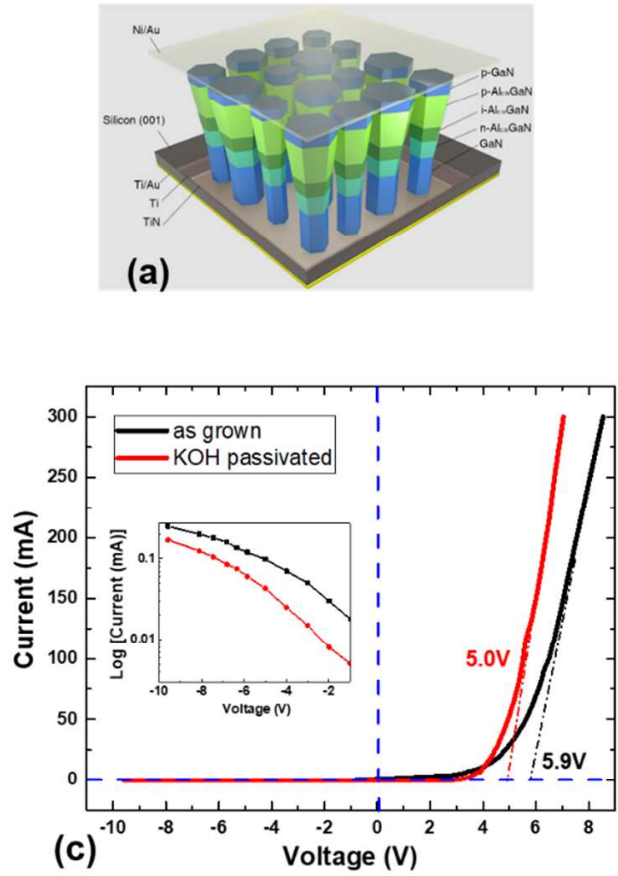
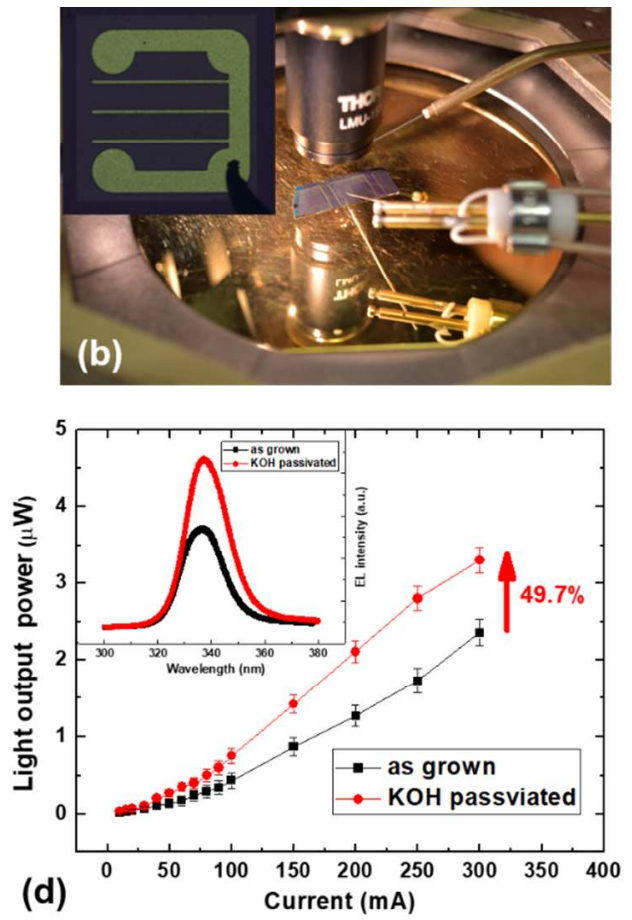

Figure 4. (a) 3D schematic depiction of the fabricated $300 \times 300 \mu \mathrm{m}^{2}$ nanowire UVLED. (b) The EL measurement setup on a probe station. The inset shows the probed device in operation. (c) Current-voltage characteristics of the fabricated sample before and after passivation. The inset shows the reverse current in log scale. (d) The measured light output power as a function of the 
injection current. The inset of (d) shows the EL spectra at an injection current of $300 \mathrm{~mA}$ before and after passivation at room temperature.

After the passivation, the nanowire UVLEDs were immediately fabricated with a chip size of $300 \times 300 \mu \mathrm{m}^{2}$. Fig. 4(a) shows a 3D schematic depiction of the fabricated device. To avoid the potential performance variations across the 2-inch wafer during the MBE growth, similar parts of the wafer were used for comparison. Fig. 4(b) shows the probe station and EL measurement setup. Fig. 4 (c) shows the representative current-voltage (I-V) characteristics for UVLEDs. A slight decrease $(\sim 0.9 \mathrm{~V})$ in the turn-on voltage and a small reduction in the sheet resistance were observed by comparing the fabricated devices before and after $\mathrm{KOH}$ passivation, revealing an improvement in the contact resistivity of $\mathrm{p}-\mathrm{GaN}$ after the $\mathrm{KOH}$ solution treatment. It was found that the lowest contact resistivity can be achieved by the surface treatment of the p-type GaN thin film using a $\mathrm{KOH}$ solution prior to the metal deposition due to their success in removing the surface oxides formed on p-type GaN during epitaxial growth. ${ }^{29,30}$ Such oxides play a vital role in inhibiting the hole transport from metal to p-type GaN. Reducing the turn-on voltage is very important for improving the overall LED performance because it is correlated with the device reliability and power consumption. High turn-on voltage is commonly believed to be linked with high contact resistivity and low-conductive films. Additionally, a smaller leakage current was observed in the device after the $\mathrm{KOH}$ treatment, as shown in the inset of Fig. 4(c), indicating that the $\mathrm{KOH}$ may have removed surface defects which could act as leakage current paths.

Fig. 4(d) shows the light output power as a function of the injection current of the studied UVLEDs at room temperature. Noticeably, the light output power of the UVLEDs shows a great enhancement (49.7\%) after KOH passivation. The inset of Fig. 4(d) shows the EL signal with a maximum peak wavelength of 336 and $338 \mathrm{~nm}$-without parasitic emission-for the studied LEDs before and after passivation, respectively. The full width at half maximum (FWHM) is 18.9 and $17.6 \mathrm{~nm}$ for these LEDs before and after passivation, respectively. Worth mentioning that it is important to note that the output power continuously increases as the current density increases up to $120 \mathrm{~A} / \mathrm{cm}^{2}$. This finding is similar to the results that we have reported in our earlier work, ${ }^{13}$ where we demonstrated that the AlGaN nanowire UVLEDs exhibited no efficiency droop at a high injected current density as the nanowires were grown on metal 
substrates, which have better heat dissipation. In contrast, we observed a significant efficiency degradation in the nanowires grown on Si substrate, ${ }^{31}$ confirming the feasibility of using metal substrate for growing AlGaN nanowire-based emitters. However, the output power of our fabricated device was still low. To improve the device performance, there are many approaches such as (1) one can use $\mathrm{AlGaN} / \mathrm{AlGaN}$ multiple quantum well structures in the active region instead of using a AlGaN double heterostructure, (2) one can optimize the growth condition (nanowire dimensions and density) to further improve the quantum efficiency of the device, (3)one can use different metal substrates to improve the light extraction efficiency, e.g., Aluminum which has much higher reflectivity $(>95 \%)$ than Titanium $(>80 \%)$ in UV spetrumal range, (4) one can minimize the thickness of the bottom n-GaN layer which is UV-absorptive, and (5) one can apply a tunneling junction to replace the p-GaN layer to avoid its absorption. ${ }^{5}$
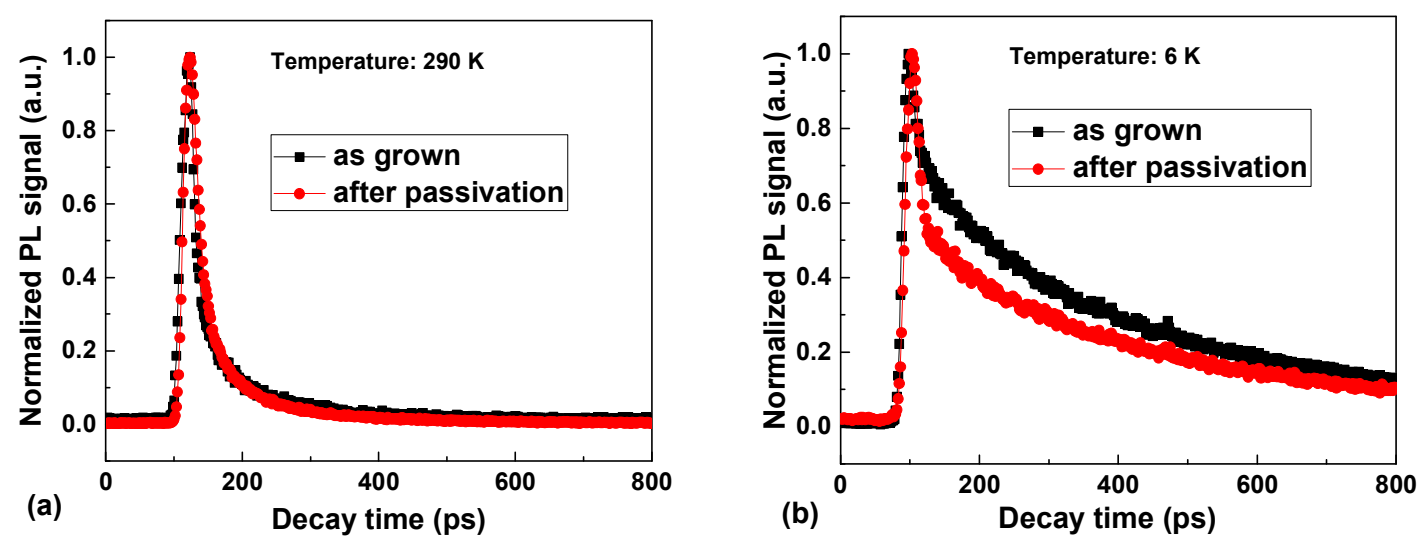

Figure 5. PL transients of as-grown and passivated AlGaN nanowires collected at (a) Roomtemperature and (b) $6 \mathrm{~K}$.

To further investigate the effects of the surface passivation on the photo-excited carrier dynamics, we also performed TRPL measurements on both as-grown and KOH-passivated AlGaN nanowires. Fig. 5 shows the low-temperature (LT) and room-temperature (RT) TRPL spectra of the major peak ( $\square 337 \mathrm{~nm}$ ) emitted from the active region. A pulse-width limited rise in the emission signal was followed by a PL decay that could be fitted fairly well with a double exponential function that is due to multiple recombination centers. ${ }^{32}$ Our study shows that the carrier lifetimes are relatively short, indicating the efficient radiative recombination of photoexcited carriers in the $\mathrm{AlGaN}$ nanowires in the absence of competition from carrier localization 
states, which is consistent with the decay profiles previously reported for MBE-grown nitrides nanowires. ${ }^{14,15}$ While the slow decay time is $\square 340$ ps at LT for as-grown nanowires, it is $\square 285$ ps for KOH-passivated nanowires. At LT, the recombination process is dominated by the radiative recombination. ${ }^{33}$ However, the role of non-radiative recombination centers is reduced but still presents. The reduced value of the slow decay time of $\mathrm{KOH}$-passivated nanowires at LT indicates a better radiative recombination process and reflects the improved quality of the nanowires. In other words, the $\mathrm{KOH}$ passivated nanowires have relatively less defect states than the as-grown ones. On the other hand, the slow decay time is $\square 108$ and $\square 125$ ps for as-grown and $\mathrm{KOH}-$ passivated nanowires at RT, respectively. The non-radiative recombination centers dominate the recombination process at RT. ${ }^{32}$ Again, the reduced density of defect states in the passivated nanowires causes the non-radiative recombination process to be slower than that of the as-grown ones. This may cause an increase in the overall carrier lifetime in the passivated nanowires. These observations provide strong evidence for the elimination of surface states via $\mathrm{KOH}$ passivation, resulting in a more efficient LED device performance.

\section{CONCLUSIONS}

In this article, we have presented an effective and environmentally-friendly method to significantly remove surface oxidation and reduce surface states in MBE-grown AlGaN nanowires by using $\mathrm{KOH}$ passivation. The STEM investigation has been used to demonstrate the almost intact nanowires after passivation. Both PL and EL results showed a significant enhancement in the optical performance of the studied UVLEDs. In particular, the optical output power is increased by about $49.7 \%$ with a better I-V characteristic in the fabricated UVLEDs emitting at $337 \mathrm{~nm}$ after $\mathrm{KOH}$ passivation. The observation of the changes of carrier lifetime before and after passivation both at room and low temperature is mainly due to the success in reducing of surface states. We anticipate that this passivation procedure will find broad practical applications in fabricating self-assembled nanowire-based high-efficient light emitting devices.

\section{AUTHOR INFORMATION \\ Corresponding Authors \\ \#boon.ooi@kaust.edu.sa, ${ }^{\#}$ xiaohang.li@kaust.edu.sa}


ACS Photonics Revised Manuscript \# ph-2017-01235t

Notes

The authors declare no competing financial interest.

\section{ACKNOWLEDGEMENTS}

We acknowledge the financial support from King Abdullah University of Science and Technology (KAUST) baseline funding, BAS/1/1614-01-01 and BAS/1/1664-01-01. Also, BSO, TKN, MKS and BJ gratefully acknowledge funding support from King Abdulaziz City for Science and Technology, grant no. KACST TIC R2-FP-008. The time-resolved PL measurements have been carried out and analyzed by M. M. Muhammed and I. S. Roqan.

\section{REFERENCES}

(1) Takano, T.; Mino, T.; Sakai, J.; Noguchi, N.; Tsubaki, K; Hirayama, H. Deep-ultraviolet light-emitting diodes with external quantum efficiency higher than $20 \%$ at $275 \mathrm{~nm}$ achieved by improving light-extraction efficiency. Appl. Phys. Express 2017, 10, 031002.

(2) Sun, X.; Zhang, Z.; Chaaban, A., Ng, T. K.; Shen, C.; Chen, R.; Yan, J.; Sun, H.; Li, X.; Wang, J.; Li, J.; Alouini, M.-S.; Ooi, B. S. 71-Mbit/s ultraviolet-B LED communication link based on 8-QAM-OFDM modulation. Opt. Express 2017, 25, pp 23267-23274.

(3) Sun, H.; Yin, J.; Pecora E F; Dal Negro L; Paiella R; Moustakas, T. D. Deep-Ultraviolet Emitting AlGaN Multiple Quantum Well Graded-Index Separate-Confinement Heterostructures Grown by MBE on SiC Substrates. IEEE Photon.J. 2017, 9, pp 1-9.

(4) Sun, H.; Moustakas, T. D. UV emitters based on an AlGaN p-n junction in the form of graded-index separate confinement heterostructure. Appl. Phys. Express 2013, 7, 012104.

(5) Zhang, Y.; Krishnamoorthy, S.; Akyol, F.; Johnson, J. M.; Allerman, A. A.; Moseley, M. W.; Armstrong, A. M.; Hwang, J.; Rajan, S. Reflective metal/semiconductor tunnel junctions for hole injection in AlGaN UV LEDs. Appl. Phys. Letters 2017, 111, 051104.

(6) Liu, C.; Ooi, Y. K.; Islam, S. M.; Verma, J.; Xing, H.; Jena, D.; Zhang, J. Physics and polarization characteristics of $298 \mathrm{~nm}$ AlN-delta-GaN quantum well ultraviolet light-emitting diodes. Appl. Phys. Lett. 2017, 110, 071103.

(7) Narukawa, Y.; Ichikawa, M.; Sanga, D.; Sano, M.; Mukai T. White light emitting diodes with super-high luminous efficacy. J. Phys. D: Appl. Phys. 2010, 43, 354002.

(8) Zhao, S.; Mi, Z. Recent Advances on p-Type III-Nitride Nanowires by Molecular Beam Epitaxy. Crystals 2017, $7,268$.

(9) Li, K. H.; Liu, X.; Wang, Q.; Zhao, S.; Mi, Z. Ultralow-threshold electrically injected AlGaN nanowire ultraviolet lasers on Si operating at low temperature. Nat. Nanotechnol. 2015, 10, 140. 
(10) Tran, N. H.; Le, B. H.; Zhao, S.; Mi, Z. On the mechanism of highly efficient p-type conduction of Mg-doped ultra-wide-bandgap AIN nanostructures. Appl. Phys. Lett. 2017, 110, 032102.

(11) Djavid, M.; Mi, Z. Enhancing the light extraction efficiency of AlGaN deep ultraviolet light emitting diodes by using nanowire structures. Appl. Phys. Lett. 2016, 108, 051102.

(12) Zhao, S.; Liu, X.; Wu, Y.; Mi, Z. An electrically pumped $239 \mathrm{~nm}$ AlGaN nanowire laser operating at room temperature. Appl. Phys. Lett. 2016, 109, 191106.

(13) Janjua, B.; Sun, H.; Zhao, C.; Anjum, D. H.; Priante, D.; Alhamoud, A. A., Wu, F.; Li, X.; Albadri, A. M.; Alyamani, A. Y.; El-Desouki, M. M.; Ng, T. K.; Ooi, B. S. Droop-free AlxGa1-xN/AlyGa1-yN quantum-disks-innanowires ultraviolet LED emitting at $337 \mathrm{~nm}$ on metal/silicon substrates. Opt. Express 2017, 25, 1381-1390.

(14) Varadhan, P.; Fu, H. C.; Priante, D.; Retamal, J. R. D.; Zhao, C.; Ebaid, M.; Ng, T. K.; Ajia, I.; Mitra, S.; Roqan, I. S.; Ooi B. S.; He, J. H. Surface Passivation of GaN Nanowires for Enhanced Photoelectrochemical Water-Splitting. Nano Lett. 2017, 17, 1520-1528.

(15) Khan, J. I.; Adhikari, A.; Sun, J.; Priante, D.; Bose, R.; Shaheen, B. S., Ng, T. K.; Zhao, C.; Bakr, O. M.; Ooi, B. S.; Mohammed, O.F. Enhanced Optoelectronic Performance of a Passivated Nanowire-Based Device: Key Information from Real-Space Imaging Using 4D Electron Microscopy. Small 2016, 12, 2313-2320.

(16) Zhao, C.; Ng, T. K.; Prabaswara, A.; Conroy, M.; Jahangir, S.; Frost, T.; O’Connell, J.; Holmes, J. D.; Parbrook, P. J.; Bhattacharya, P.; Ooi, B. S.; An enhanced surface passivation effect in InGaN/GaN disk-in-nanowire light emitting diodes for mitigating Shockley-Read-Hall recombination. Nanoscale 2015, 7, 16658-16665.

(17) Martin, H.; Julia, W.; Luca, F.; Max, K.; Markus, D.; Sonja, M.; Anna, F. M.; Martin, S. Surface passivation and selfregulated shell growth in selective area-grown $\mathrm{GaN}-(\mathrm{Al}, \mathrm{Ga}) \mathrm{N}$ core-shell nanowires. Nanoscale 2017, 9, 7179-7188.

(18) AlOtaibi, B.; Harati, M.; Fan, S.; Zhao, S.; Nguyen, H. P.; Kibria, M. G.; Mi, Z. High efficiency photoelectrochemical water splitting and hydrogen generation using $\mathrm{GaN}$ nanowire photoelectrode. Nanotechnology 2013, 24, 175401.

(19) Chevtchenko, S. A.; Reshchikov, M. A.; Fan, Q.; Ni, X.; Moon, Y. T.; Baski, A. A.; Morkoç, H., Study of SiNx and $\mathrm{SiO} 2$ passivation of GaN surfaces. J. Appl. Phys. 2007, 101, 113709.

(20) Li, Q.; Wright, J. B.; Chow, W. W.; Luk, T. S.; Brener, I.; Lester, L. F.; Wang, G. T. Single-mode GaN nanowire lasers. Opt. Express 2012, 20, 17873-17879.

(21) Q. Li, J. B. Wright, W. W. Chow, T. S. Luk, I. Brener, L. F. Lester, and G. T. Wang, Opt. Express 2012, 20, 17873

(22) Yatabe, Z.; Asubar, J.; Hashizume, T. Insulated gate and surface passivation structures for GaN-based power transistors. J. Phys. D: Appl. Phys. 2016, 49, 393001.

(23) Pierret, A.; Bougerol, C.; Murcia-Mascaros, S.; Cros, A.; Renevier, H.; Gayral, B.; Daudin, B. Growth, structural and optical properties of AlGaN nanowires in the whole composition range. Nanotechnology 2013, 24, 115704.

(24) Prabaswara, A.; Stowe, D. J.; Janjua, B.; Ng, T. K.; Anjum, D. H.; Longo, P.; Zhao, C.; Elafandy, R. T.; Li, X.; Y. Alyamani, A.; El-Desouki, M. M.; Ooi B. S.; Spatially resolved investigation of competing nanocluster emission 
in quantum-disks-in-nanowires structure characterized by nanoscale Cathodoluminescence. J. Nanophotonics 2017, 11(2), 026015.

(25) Li, D. S.; Sumiya, M.; Fuke, S.; Yang, D.; Que, D.; Suzuki, Y.; Fukuda, Y. Selective etching of GaN polar surface in potassium hydroxide solution studied by x-ray photoelectron spectroscopy. J. Appl. Phys. 2001, 90, 4219. (26) Wang, H.; Zhai, G.; Shang, L.; Ma, S.; Jia, W.; Jia, Z.; Liang, J.; Li, X.; Xu, B. The morphologies and optical properties of three-dimensional GaN nano-cone arrays. RSC Adv. 2016, 6, 43272-43277.

(27) Paramanik, D.; Motayed, A.; King, M.; Ha, J.-Y.; Kryluk, S.; Davydov, A. V.; Talin, A. Fabrication of high quality GaN nanopillar arrays by dry and wet chemical etching. arXiv:1311.0321v3 [condmat.mtrl-sci] (2014).

(28) Fujii, T.; Gao, Y.; Sharma, R.; Hu, E. L.; DenBaars, S. P.; Nakamura, S. Increase in the extraction efficiency of GaN-based light-emitting diodes via surface roughening. Appl. Phys. Lett. 2004, 84, 855.

(29) Lee, J.-L.; Kim, J. K.; Lee, J. W.; Park, Y. J.; Kim, T. Effect of surface treatment by KOH solution on ohmic contact formation of p-type GaN. Solid-State Electron. 1999, 43, 435-438.

(30) Sun, J.; Rickert, K. A.; Redwing, J. M.; Ellis, A. B.; Himpsel, F. J.; Kuech, T. F. p-GaN surface treatments for metal contacts. Appl. Phys. Lett. 2000, 76, 415.

(31) Janjua, B.; Sun, H.; Zhao, C.; Anjum, D. H.; Wu, F.; Alhamoud, A. A.; Li, X.; Albadri, A. M.; Alyamani, A. Y.; El-Desouki, M. M.; Ng, T. K.; Ooi, B. S. Self-planarized quantum-disks-in-nanowires ultraviolet-B emitters utilizing pendeo-epitaxy. Nanoscale 2017, 9, 7805-7813.

(32) Rao, MSR.; Okada, T. ZnO nanocrystals and allied materials. Springer India, New Delhi: 2014, 209-211.

(33) Deshpande, S.; Frost, T.; Yan, L.; Jahangir, S.; Hazari, A.; Liu, X.; Mirecki-Millunchick, J.; Mi, Z.;

Bhattacharya, P. Nano Lett. 2015, 15, 1647-1653 


\section{For Table of Contents Use Only (TOC)}

\section{Surface-Passivated AlGaN Nanowires for Enhanced \\ Luminescence of Ultraviolet Light Emitting Diodes}

Haiding Sun1, Mohammad Khaled Shakfa2, Mufasila Mumthaz Muhammed3, Bilal Janjua2, Kuang-Hui Li1, Ronghui Lin1, Tien Khee Ng2, Iman S. Roqan3, Boon S. Ooi2\#, Xiaohang Li1, \#\#

1 King Abdullah University of Science \& Technology (KAUST), Computer, Electrical, and Mathematical Sciences and Engineering Division, Advanced Semiconductor Laboratory, Thuwal 23955-6900, Saudi Arabia

2 King Abdullah University of Science \& Technology (KAUST), Computer, Electrical, and Mathematical Sciences and Engineering Division, Photonics Laboratory, Thuwal 23955-6900, Saudi Arabia

3 King Abdullah University of Science and Technology (KAUST), Physical Sciences and Engineering Division, Spectroscopy and Semiconductor Research Laboratory, Thuwal 23955, Saudi Arabia 
ACS Photonics Revised Manuscript \# ph-2017-01235t

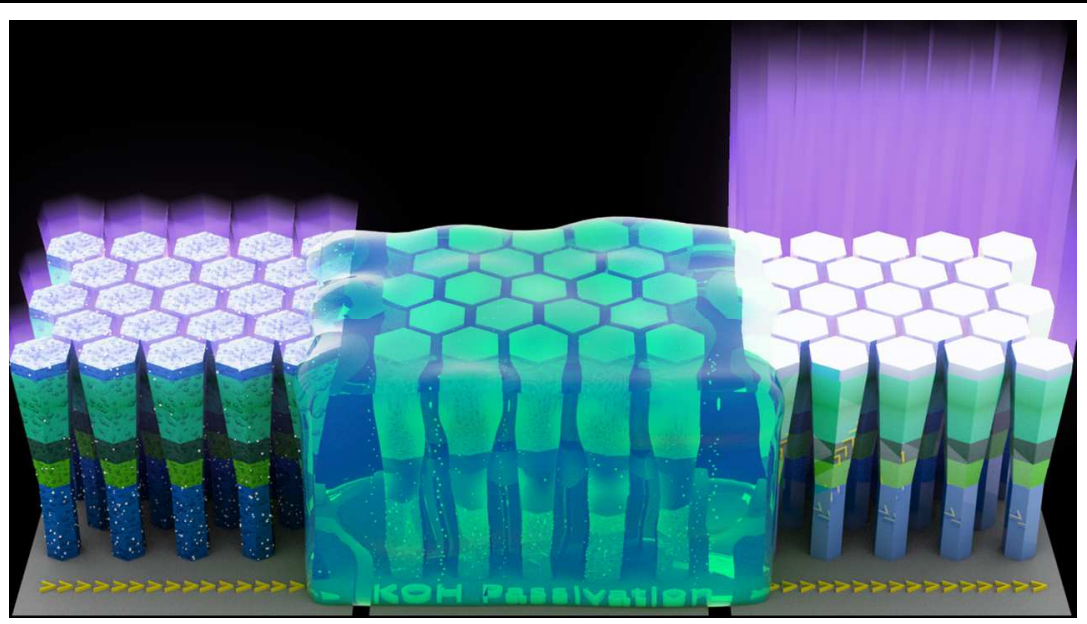

An effective and environmentally-friendly method to significantly remove surface oxidation and reduce surface states in $\mathrm{MBE}$-grown $\mathrm{AlGaN}$ nanowires by using $\mathrm{KOH}$ solution and thus remarkably enhance the ultraviolet light output power. 

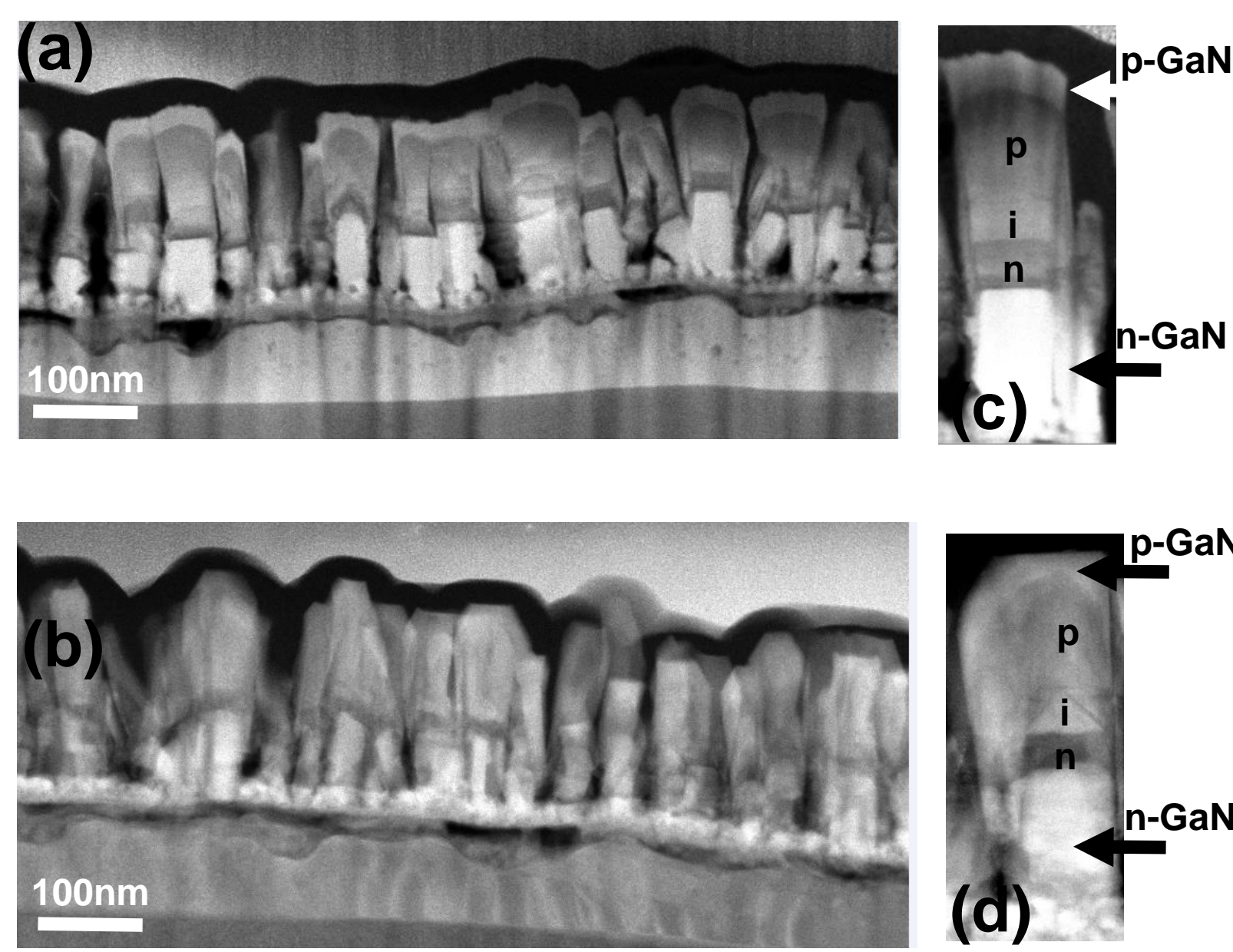
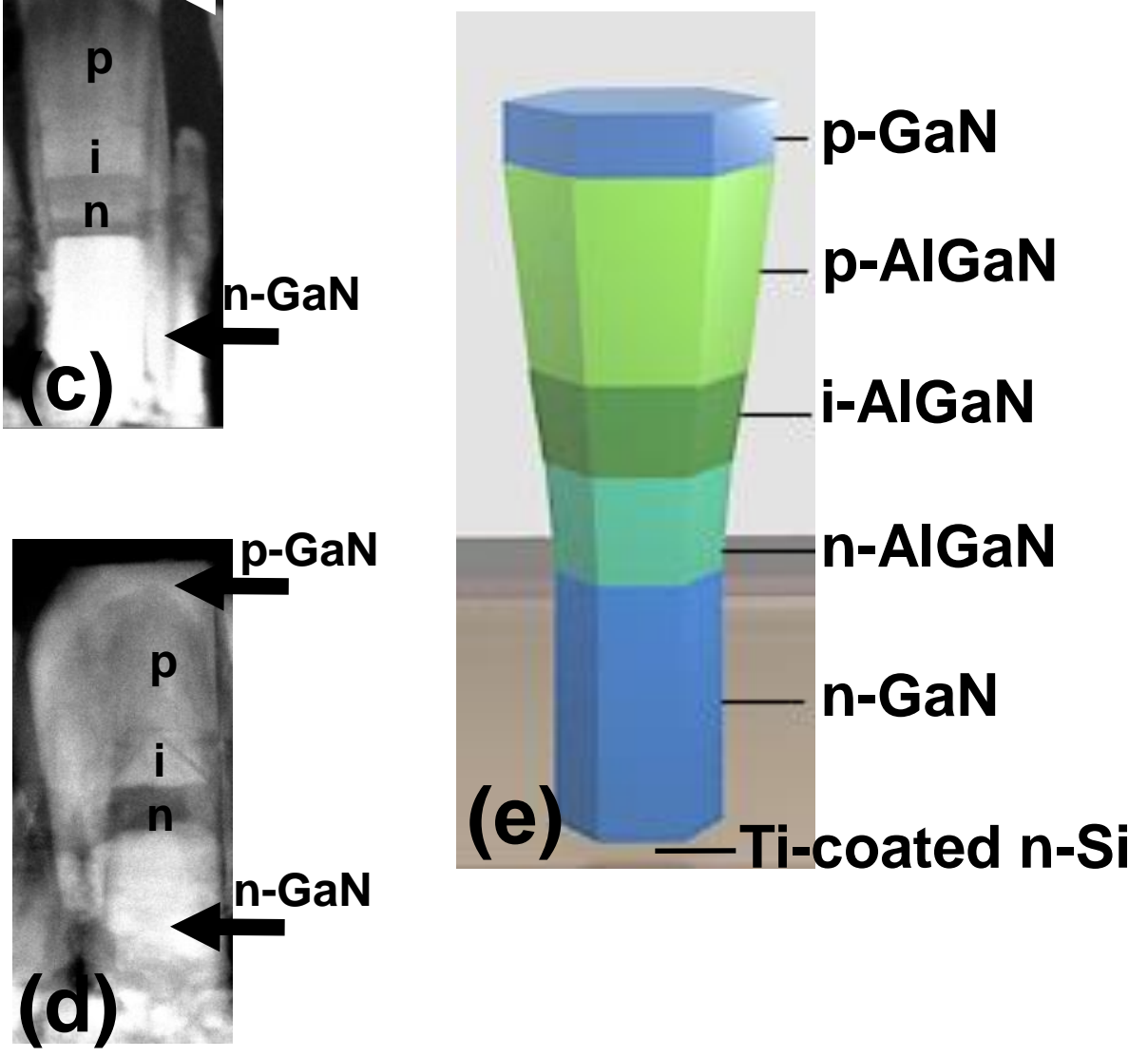
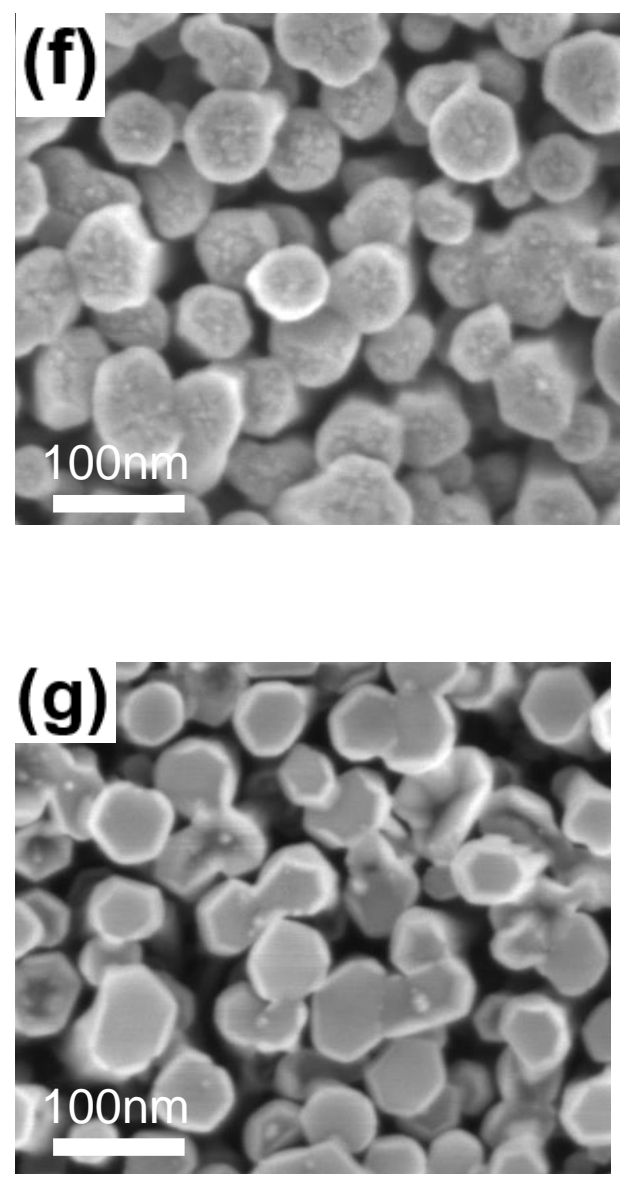


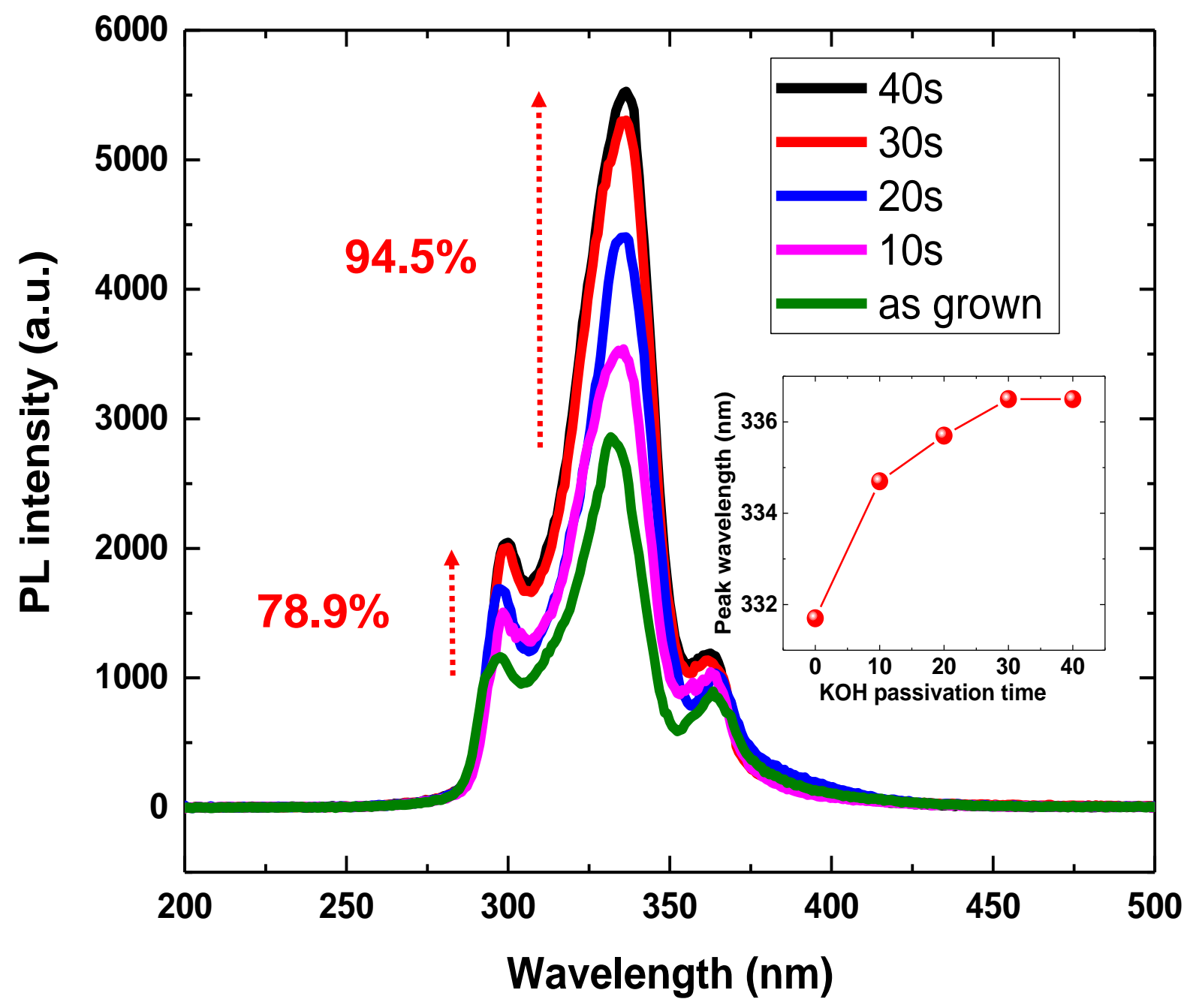



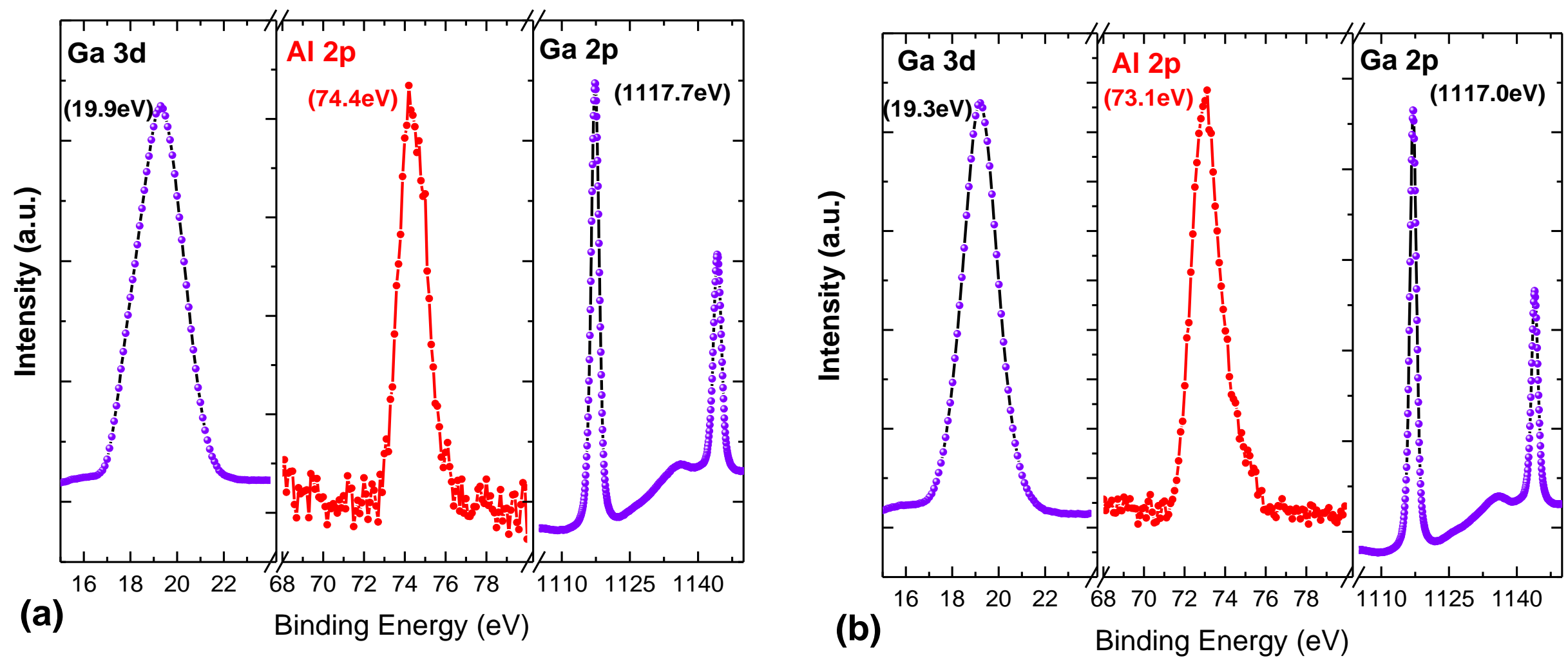

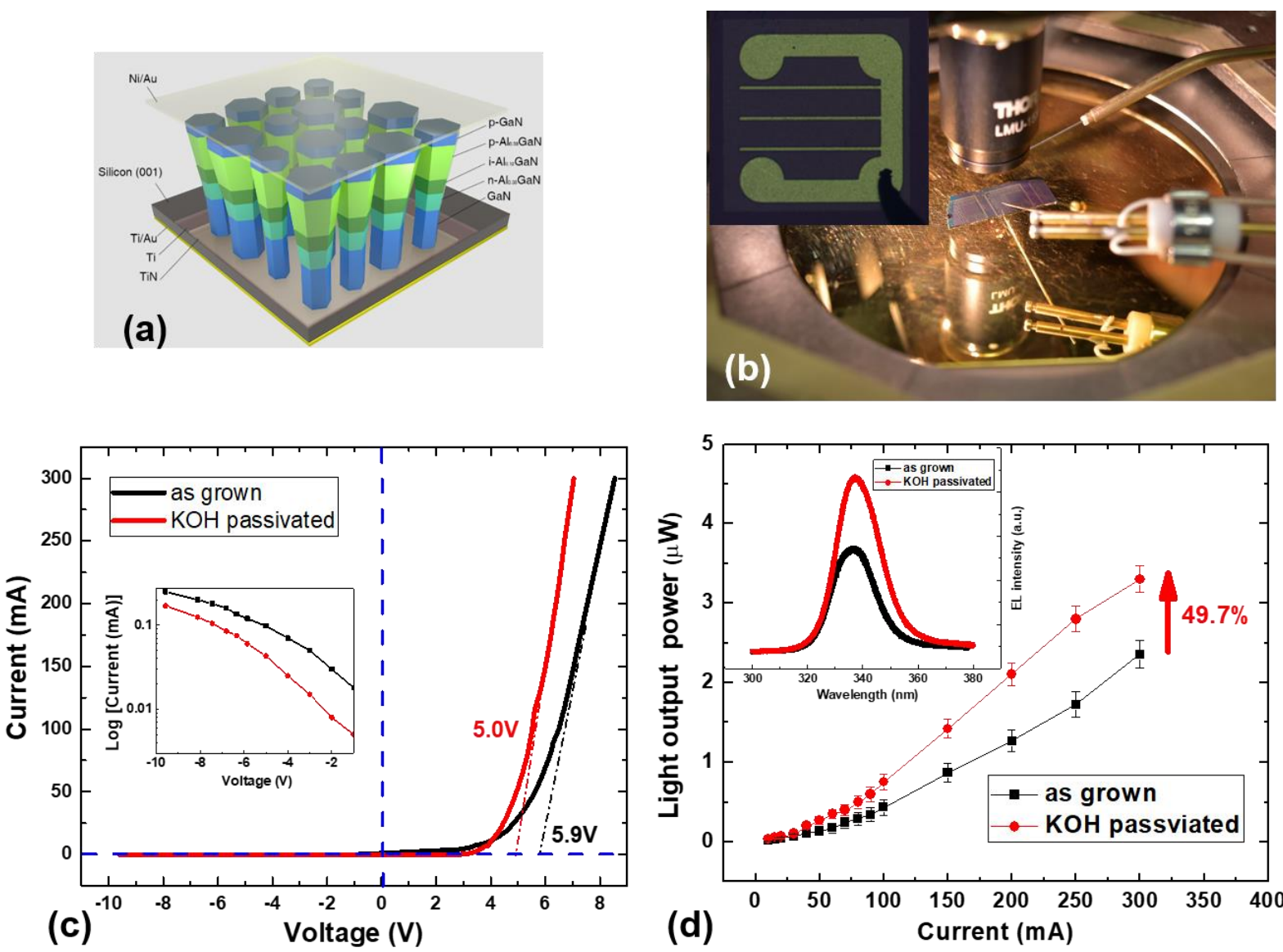

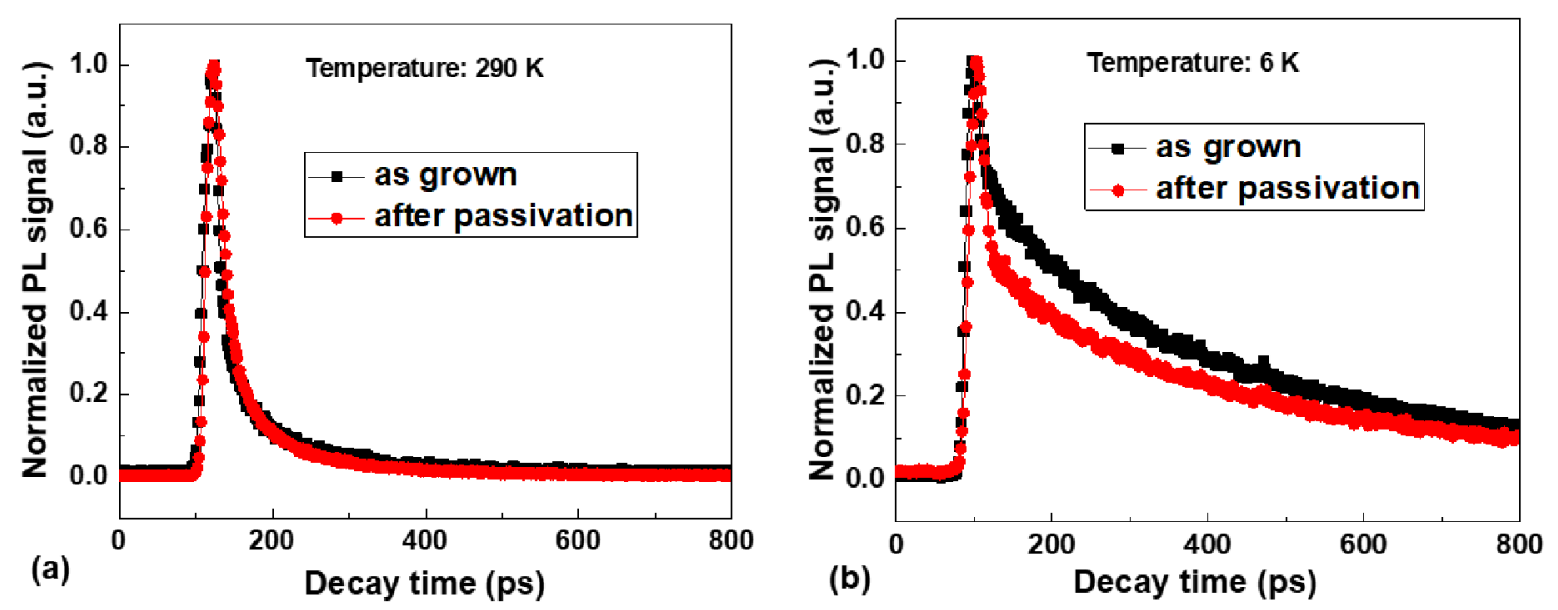